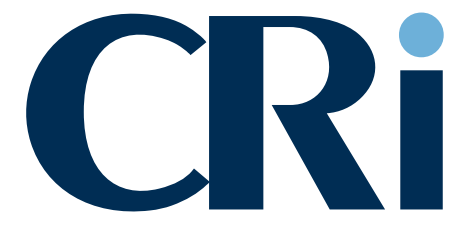

Issue 4

15 August 2007

A Journal

P. $97-128$

PVSt 52028 \title{
of Information Law and Technology
}

\section{Articles}

Susan Hall - Legal Conundrums Regarding the Use of ICT in Healthcare

Wolfgang Kilian - The UN-Convention on the Use of

E-Communications in International Contracts

\section{Case Law}

USA: Copyright Liability of Search Engines Perfect 10, Inc. v. Amazon.com

Council of Europe: Monitoring of Phone, E-Mail and Internet at Work Copland v. the United Kingdom

USA: ISP Liability For Third Party Contents Perfect 10, Inc.

v. CCBill LLC et al. with remarks by Band

EPO: Technical Character of Business Research Method Duns Licensing Associates, LP.

\section{Updates}

Probir Roy Chowdhury - India: Measuring Up Against Cyber Crime

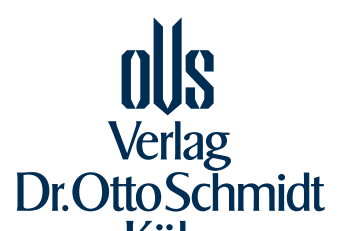

Köln 


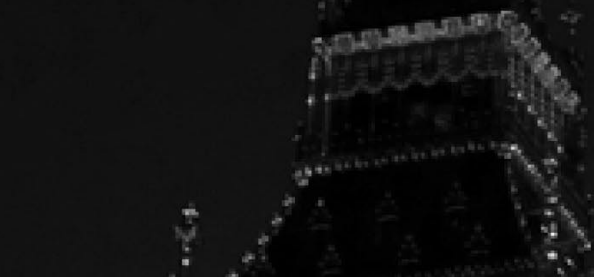

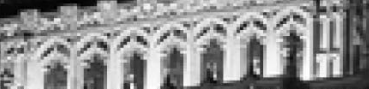

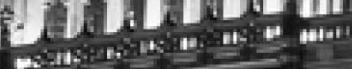
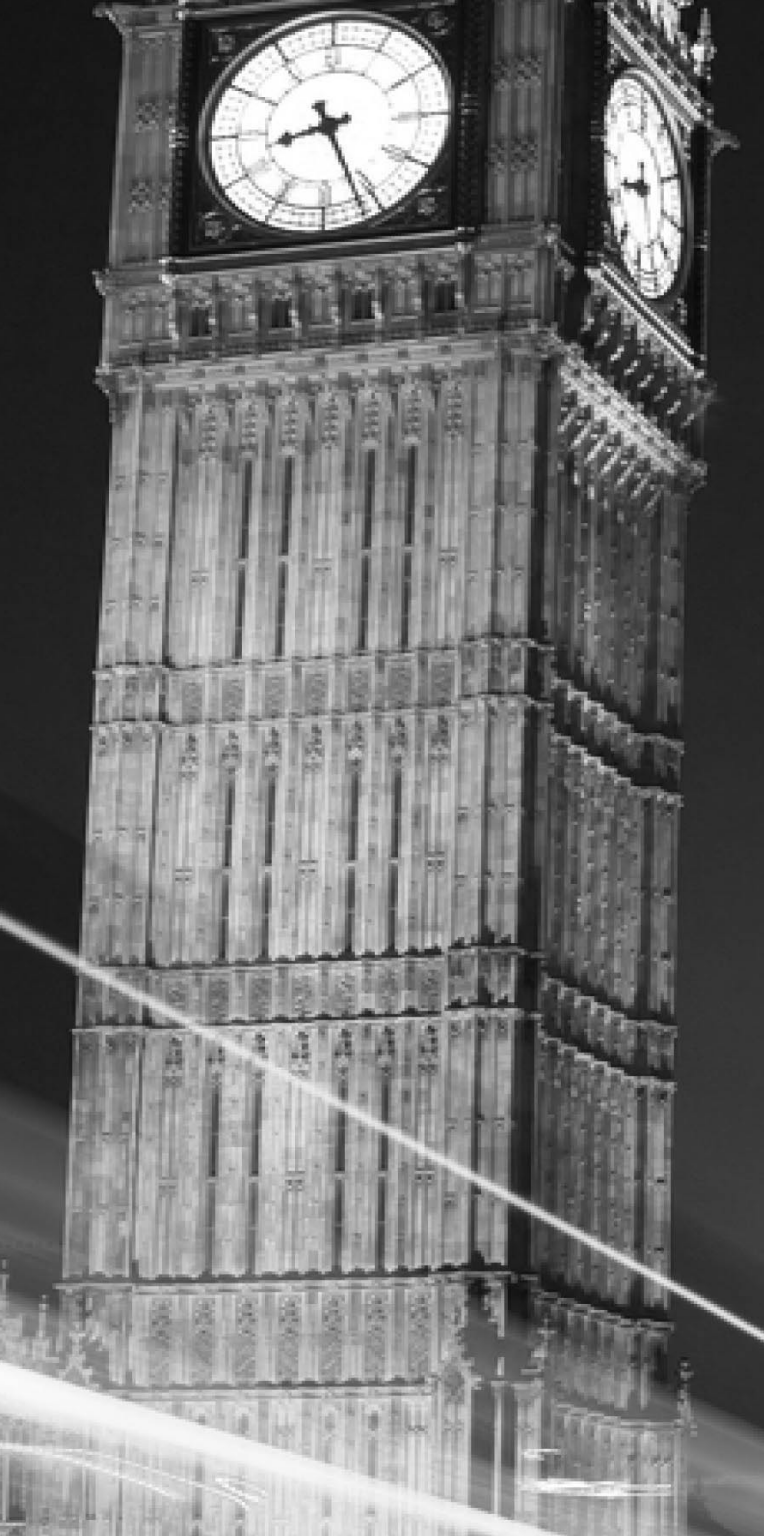

\section{Conference Co-Chairs:}

Alexander Blumrosen

Bernard Hertz Bejot, Paris

Kiran Sandford

Mishcon de Reya, London

Enrique Batalla

Batalla Abogados, Madrid

and President of ITechLaw
ITechLaw Program Committee Chair: John Beardwood

Fasken Martineau DuMoulin LLP, Toronto

Merchant Taylors' Hall November 1-2, 2007 LONDON i. if

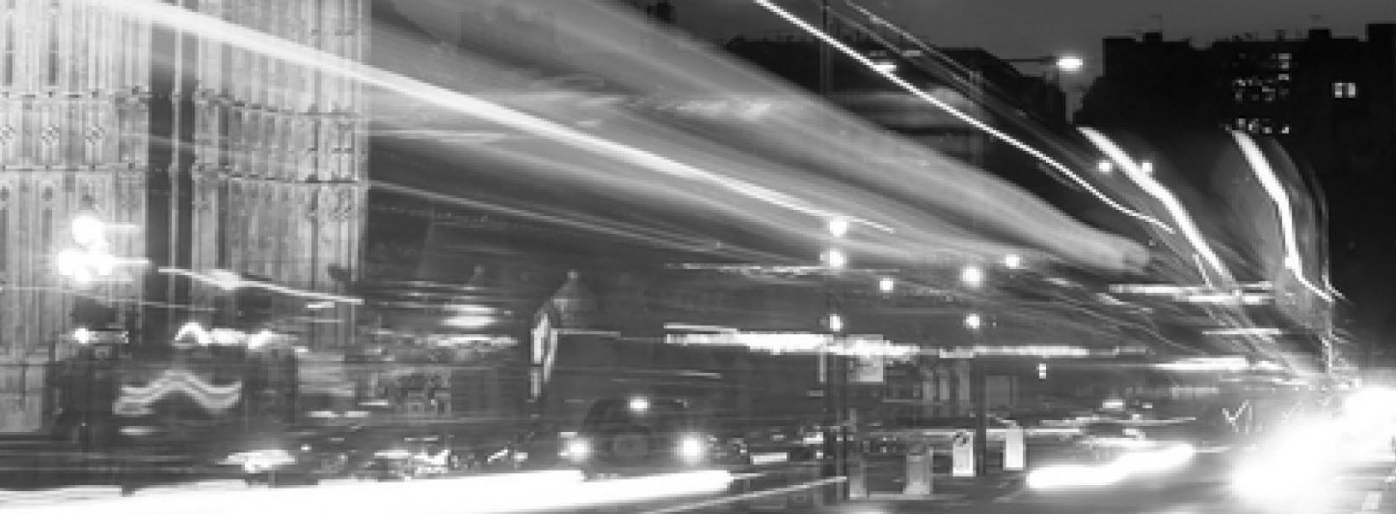

\section{presents \\ INIERNATIONAL TECHNOLOGY LAW ASSOCIATION}

European onference 
New at www.cr-international.com:

Agreement of 23 July 2007 between EU and US on PNR Data

\section{Computer Law Review Internationa1}

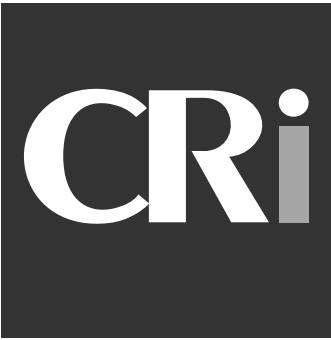

A Journal of Information Law and Technology

8th Year · Issue 4/2007 · Pages 97-128

\section{Editorial Board}

Prof. Dr. Thomas Dreier, M.C.J.

University of Karlsruhe

Dr. Jens-L. Gaster, principal administrator, Brussels

RA Thomas Heymann, Frankfurt/M.

Prof. Dr. Michael Lehmann, Dipl.-Kfm,

Max-Planck-Institute and University of Munich

Prof. Raymond T. Nimmer, University of Houston

Attorney at Law Holly K. Towle, J.D., Seattle

Attorney at Law Thomas Vinje, Brussels

\section{Articles}

Susan Hall - Legal Conundrums Regarding the Use of ICT in Healthcare A legal and contractual analysis of current applications of ICT in the healthcare field

Wolfgang Kilian - The UN-Convention on the Use of E-Communications in International Contracts Applicability, scope and potential impact of the emerging pendant to CISG

\section{Case Law}

Attorney at Law Sakari Aalto (Finland)

Attorney at Law Jonathan Band (USA)

Prof. Dr. Janusz Barta (Poland)

Abogado Enrique J. Batalla (Spain)

John P. Beardwood (Canada)

Prof. Dr. Jon Bing (Norway)

DDr. Walter Blocher (Austria)

Prof Peter Blume (Denmark)

Awocato Gabriel Cuonzo (Italy)

Dr. Jens-L. Gaster (EU)

Prof. Ysolde Gendreau (Canada)

Dr. Lucie Guibault (Canada/Netherlands)

Avocat Dr. Martin Hauser (France)

Prof. Dr. Rosa Julia-Barcelo (Spain)

Attorney at Law Charles H. Kennedy (USA)

Dr. Stanley Lai (Singapore)

Prof. Ian Lloyd (UK)

RA Prof. Dr. Michail Marinos (Greece)

Prof. Dr. Ryszard Markiewicz (Poland)

Antonio Millé (Argentina)

Ken Moon (New Zealand)

Prof. Raymond T. Nimmer (USA)

Advogado Manuel Oehen Mendes (Portugal)

Prof. Jerome Reichman (USA)

Luis C. Schmidt (Mexico)

Harry Small (UK)

Prof. Alain Strowel (Belgium)

Awocato Pietro Tamburrini (Italy)

Attorney at Law Thomas Vinje (USA, EU)

Prof. Coenraad J. Visser (South Africa)

Prof. Dr. Rolf H. Weber (Switzerland)

JT. Westermeier (USA)

Neil J. Wilkof (Israel)

Jamie Wodetzki (Australia)
USA: No Secondary Liability of Credit Card Services For Third Party Contents Perfect 10, Inc. v. Visa International Service Association et al. - Court of Appeals for the 9th Circuit - Decision of 3 July 2007106

EU: Collection of VAT on Grant of UMTS Licence Hutchinson 3G UK Ltd et al. v. Commissioner of Customs and Excise - European Court of Justice - Decision of 26 June 2007

USA: Copyright Liability of Search Engines Perfect 10, Inc. v. Amazon.com - Court of Appeal for the 9th Circuit - Decision of 16 May 2007

Council of Europe: Monitoring of Phone, E-Mail and Internet at Work Copland v. the United Kingdom - European Court of Human Rights Decision of 3 April 2007

USA: ISP Liability For Third Party Contents Perfect 10, Inc. v. CCBill LLC et al. - Court of Appeals for the 9th Circuit - Decision of 29 March 2007 with remarks by Band

EPO: Technical Character of Business Research Method Duns Licensing Associates, L.P. [T-0154/04 - 3.5.2001] - Technical Board of Appeal - Decision of 15 November 2006

\section{Updates}

Probir Roy Chowdhury - India: Measuring Up Against Cyber Crime

- About the Authors

- Masthead

- CRi-Reader Services 

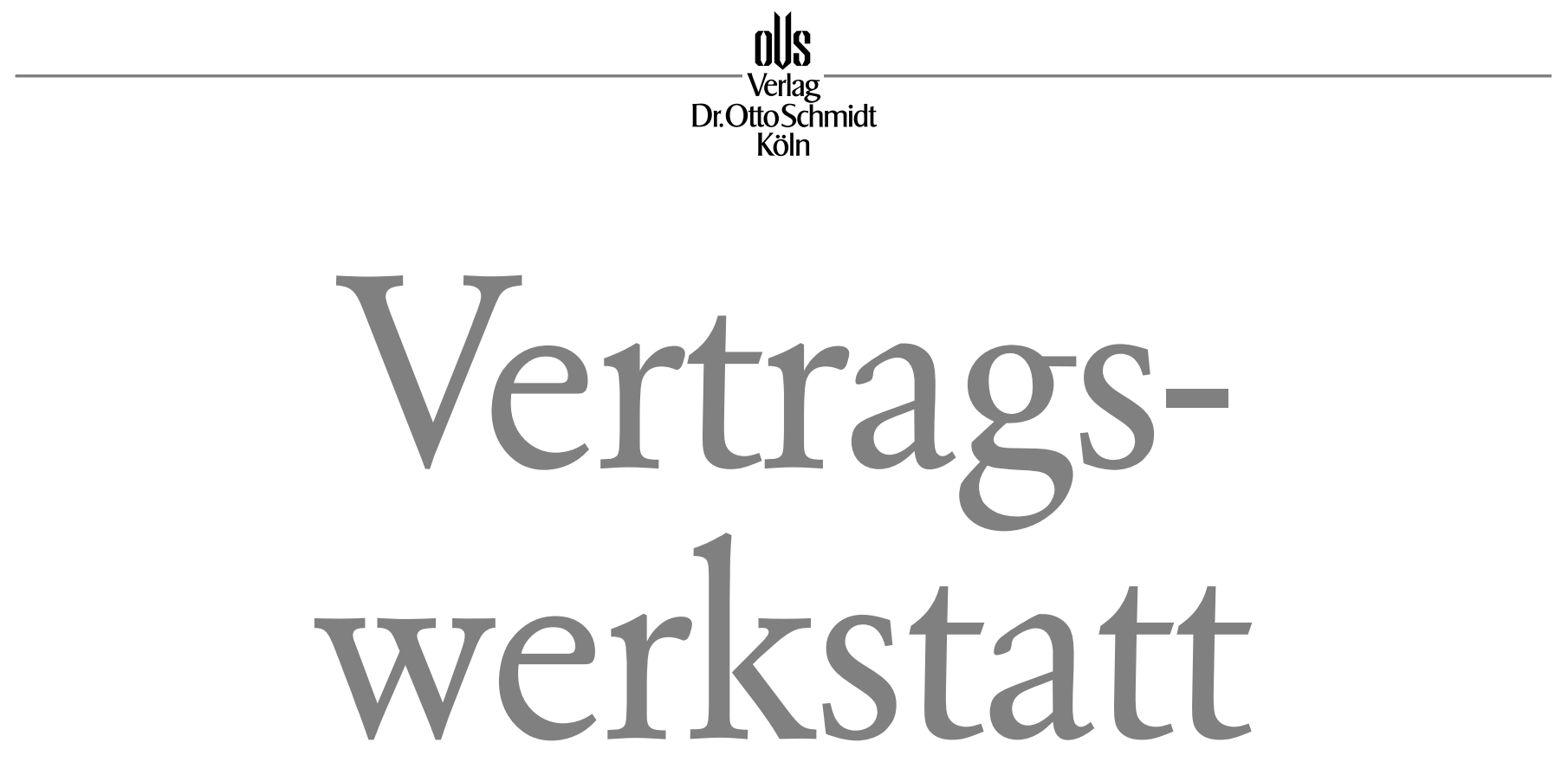

Dieses renommierte Handbuch zeigt Ihnen, wie Sie selbst schwierigste Vertragswerke fehlerlos entwerfen, verhandeln und systematisch zum gewünschten Erfolg führen. Nach einem modularen Gestaltungssystem, das auf nur zwei Grundmustern von Kontrakten basiert.

Die Vorteile dieses standardisierten Vertragsdesigns liegen auf der Hand: Einheitliche und überschaubare Grundstruktur all Ihrer Regelungswerke. Mehr Übersicht und Transparenz für Sie und Ihre Mandanten. Höhere Effizienz und niedrigere Fehlerquote. Von der Planung über die Verhandlung bis zur Abwicklung und Kontrolle Ihrer Verträge.

Neben den vielen Übersichten, Beispielen und Formulierungsvorschlägen für einzelne Klauseln geben die Autoren Ihnen auch

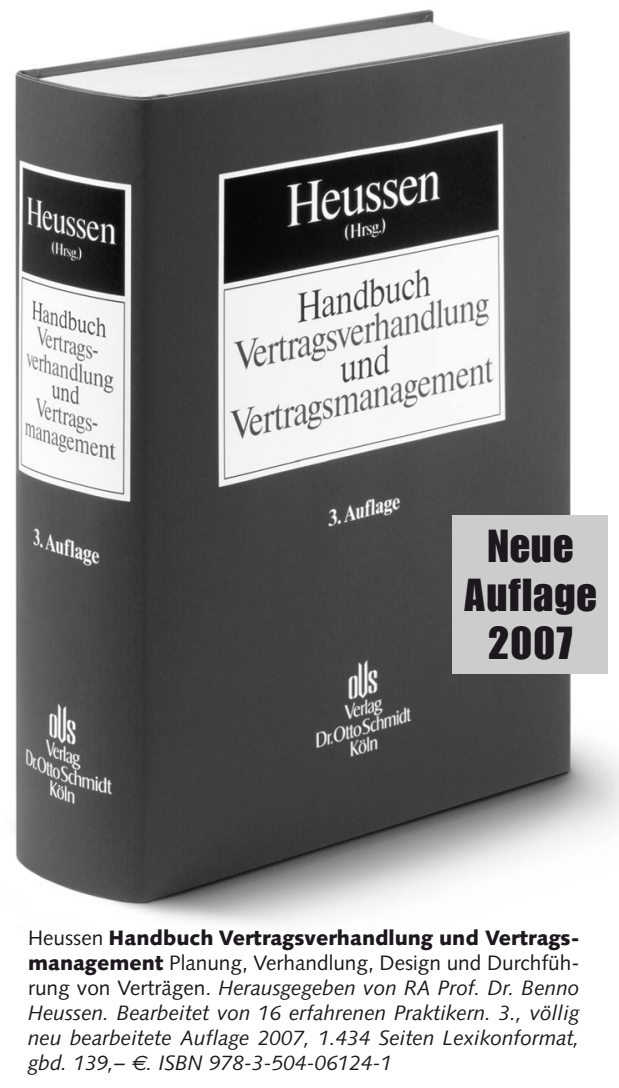

detaillierte Checklisten von kompletten Verträgen für Ihre rechtsgestaltende Tagesarbeit an die Hand.

Die neue Auflage beschäftigt sich ausführlich mit dem Verhandeln im Ausland - Japan, China, USA, Brasilien und Russland -, das in Zeiten der Globalisierung mehr und mehr an Bedeutung gewinnt. Ebenfalls neu: Qualitätsmanagement von Vertragsprojekten. Der Teil des Buches, der Sie über die typischen Fehler in der Zusammenarbeit von Managern und Beratern aufklärt und Ihnen mit Sicherheit hilft, Ihre Arbeit noch weiter zu optimieren.

Heussen, Handbuch Vertragsverhandlung und Vertragsmanagement. Für eine Leseprobe heißen wir Sie jederzeit herzlich willkommen bei www.otto-schmidt.de

Bestellschein ausfüllen und faxen (02 21) 937 38-943 옹

X. Ja, ich bestelle mit 14-tägigem Rückgaberecht Heussen Handbuch Vertragsverhandlung und Vertragsmanagement 3. Auflage, gbd. 139,-€ plus Versandkosten. ISBN 978-3-504-06124-1

\begin{tabular}{lcccc}
\hline Name & Straße & Ort & & \\
& & & \\
\hline Telefon & Fax & Datum & Unterschrift
\end{tabular}

